\title{
Linear Grain Growth Kinetics and Rotation in Nanocrystalline Ni
}

\author{
Diana Farkas, Som Mohanty, and Joshua Monk \\ Department of Materials Science and Engineering, Virginia Tech, Blacksburg, Virginia, USA
}

(Received 11 July 2006; published 18 April 2007)

\begin{abstract}
We report three-dimensional atomistic molecular dynamics studies of grain growth kinetics in nanocrystalline Ni. The results show the grain size increasing linearly with time, contrary to the square root of the time kinetics observed in coarse-grained structures. The average grain boundary energy per unit area decreases simultaneously with the decrease in total grain boundary area associated with grain growth. The average mobility of the boundaries increases as the grain size increases. The results can be explained by a model that considers a size effect in the boundary mobility.
\end{abstract}

The properties of nanocrystalline metals have been investigated using computational methods in recent years [18]. Molecular dynamics has been utilized in this kind of simulation, with the limitation of the extremely fast strain rates that are imposed by the computational resources. The applications of nanocrystalline materials are limited by the thermal stability, and the kinetics of thermal grain growth are critical for the structural stability of these materials. Both experimental and theoretical studies [9-12] have pointed out that grain growth kinetics in nanocrystalline materials may be different from that in coarse-grained materials. The nanoscale effects in the kinetics can arise from a variety of factors, such as the role of triple junctions or grain rotation.

In this Letter, we report simulation studies of grain growth kinetics in nanocrystalline $\mathrm{Ni}$ carried as a function of temperature. We study the grain growth process by monitoring the evolution of individual grains and the position of individual grain boundaries, and we perform a statistical analysis of the overall microstructural evolution. The simulations were performed using a conventional molecular dynamics (MD) algorithm. The interaction between the atoms was modeled using an embedded-atom method potential developed by Voter and Chen for nickel [13]. The sample was prepared using a Voronoi construction with an initial average grain size of $4 \mathrm{~nm}[5,6]$. The initial nickel cube contained 15 grains and 100000 atoms. The code used to run the MD tensile tests is LAMMPS, developed by Plimpton [14]. The sample was first equilibrated for 300 ps with periodicity in all directions and a temperature of $300 \mathrm{~K}$. This process ensured a stable grain boundary structure [15]. After the initial relaxation, heat treatments were performed at temperatures from 900 to $1450 \mathrm{~K}$. As the treatment proceeds, snapshots of the sample were created for visualization and analysis of the grain growth process. Figure 1 shows the microstructure evolution in a slice of the three-dimensional sample after $500 \mathrm{ps,}$ at $1300 \mathrm{~K}$. The various grains are color coded, and individual atoms are not shown for clarity. The position of the grain boundaries in the sample was determined from an analysis of the continuity of the crystallographic orienta- tion. The position of individual boundaries in slices such as that shown in Fig. 1 was monitored as a function of time. Figure 2 shows an example of the movement of one particular boundary in the sample as a function of time for various temperatures. The results show that the velocity of the grain boundary is maintained mostly constant, and the values of the grain boundary velocity for each grain boundary in the sample at each temperature can be extracted from this analysis. We found that the velocity of individual grain boundaries varied widely across the sample. For each temperature, a statistical analysis of the grain boundary velocities was performed. The results for the treatment at $1300 \mathrm{~K}$ are shown in Fig. 3. Table I shows the average grain boundary velocities and corresponding standard deviations obtained for each temperature. Typically, the standard deviation observed at each temperature is around $30 \%$ of the average velocity. We estimate the error in the average velocities to be $2 \%-5 \%$. In addition to monitoring the position of individual boundaries, the average grain size was obtained as a function of time using the standard intersection technique normally used in experimental studies. For this analysis, intersections were counted in a total of 30 sections of the sample including 10 sections perpendicular to each of the spatial directions. Using this statistical procedure, we obtain average grain sizes with an estimated $2 \%$ accuracy. The results are shown in Fig. 4 for the treatment at $1300 \mathrm{~K}$. This analysis was
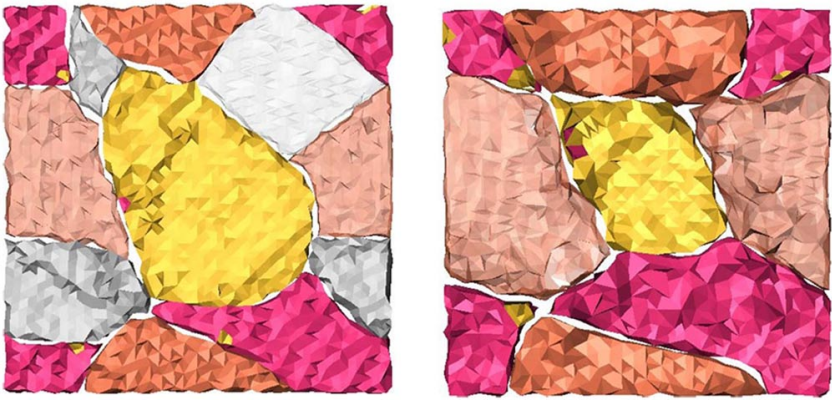

FIG. 1 (color online). Microstructure evolution after $500 \mathrm{ps}$ at $1300 \mathrm{~K}$. The grains are identified according to the continuity of crystallographic orientation. 


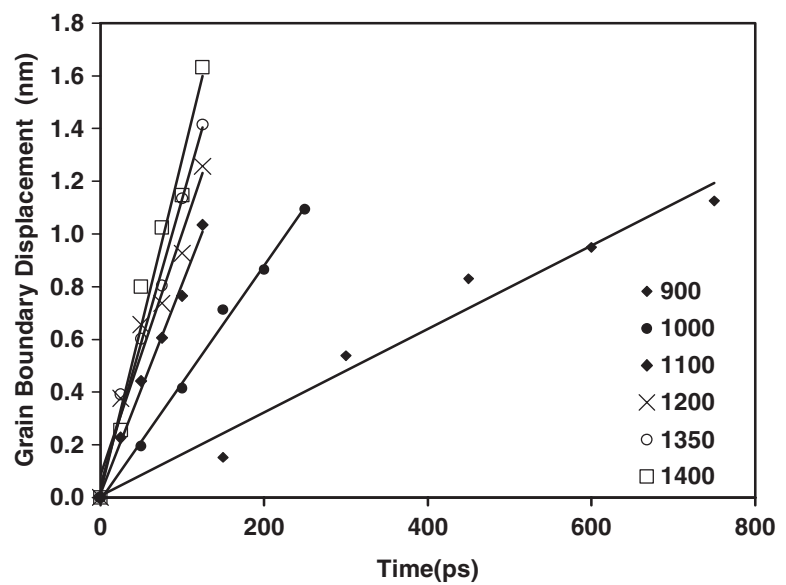

FIG. 2. Position of a particular grain boundary as a function of time for various temperatures.

repeated for each temperature. In all cases, linear grain growth was obtained. We note that, in this process, the number of grains has decreased to about half the original number, and therefore this does not represent an initial transient regime.

To complete the analysis of the observed grain growth process, we followed the energy evolution of the samples as a function of time for various temperatures. As an example, Fig. 5 shows the results for $1300 \mathrm{~K}$. In this figure, the average potential energy per atom in the sample is plotted, and we observe that the energy per atom in the sample $E$ decreases as function of time with a square root of the time dependence $E=E_{0}-K t^{1 / 2}$, where $E_{0}$ is the average (temperature-dependent) potential energy per atom in the initial equilibrated structure at $t=0$, and $K$ is a temperature-dependent constant. The values of the constant $K$ obtained from this analysis can be plotted in Arrhenius form to obtain the activation energy for the process. Figure 6 shows these results together with the Arrhenius behavior of the average grain boundary veloc-

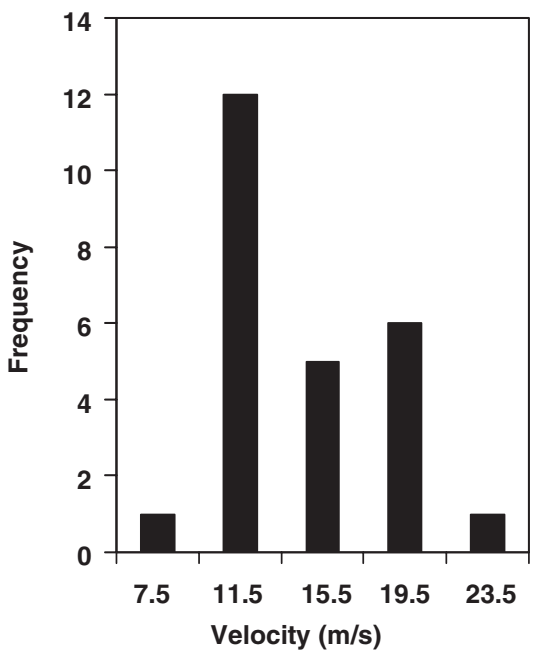

FIG. 3. Histogram of grain boundary velocities at $1300 \mathrm{~K}$.
TABLE I. Average grain boundary velocities and standard deviations for various annealing temperatures.

\begin{tabular}{ccc}
\hline \hline $\begin{array}{c}\text { Temperature } \\
(\mathrm{K})\end{array}$ & $\begin{array}{c}\text { Average velocity } \\
(\mathrm{m} / \mathrm{s})\end{array}$ & $\begin{array}{c}\text { Standard deviation } \\
(\mathrm{m} / \mathrm{s})\end{array}$ \\
\hline 900 & 1.252 & 0.394 \\
1000 & 3.610 & 1.011 \\
1100 & 8.500 & 2.041 \\
1200 & 10.711 & 2.414 \\
1300 & 12.639 & 3.627 \\
1450 & 21.146 & 7.992 \\
\hline \hline
\end{tabular}

ities in Table I. The activation energies obtained for the process using the average velocities and the energy evolution constants are nearly the same, namely, 53 and $54 \mathrm{~kJ} / \mathrm{mol}$. In a recent study of grain boundary selfdiffusion using the same potential, Mendelev et al. [16] studied a series of grain boundaries of different inclinations. They found that, except for a symmetrical tilt boundary (103), the activation energies were $48-59 \mathrm{~kJ} / \mathrm{mol}$. This excellent agreement suggests that the process observed here is indeed controlled by the same individual atomic mechanisms as grain boundary self-diffusion for nonspecial boundaries. The constant $K$ depends on temperature through the temperature dependence of grain boundary mobility as well as any changes in the average grain boundary energy in the system. Usually, in modeling grain growth, the average grain boundary energy is taken as a constant. As described below, we found that is not the case in our simulations, and this means that the relation of the constant $K$ and mobility is not straightforward, because there are two processes that contribute to the total energy decrease, namely, a decrease in total grain boundary area and a decrease of the average energy per unit area.

From our results, it is possible to obtain the average grain boundary energy per unit area by combining the results obtained for the total energy evolution of the sample and those for grain size increase. For this purpose, we have estimated the total grain boundary area in the sample as follows: If the volume of the sample $V$ is constant as the

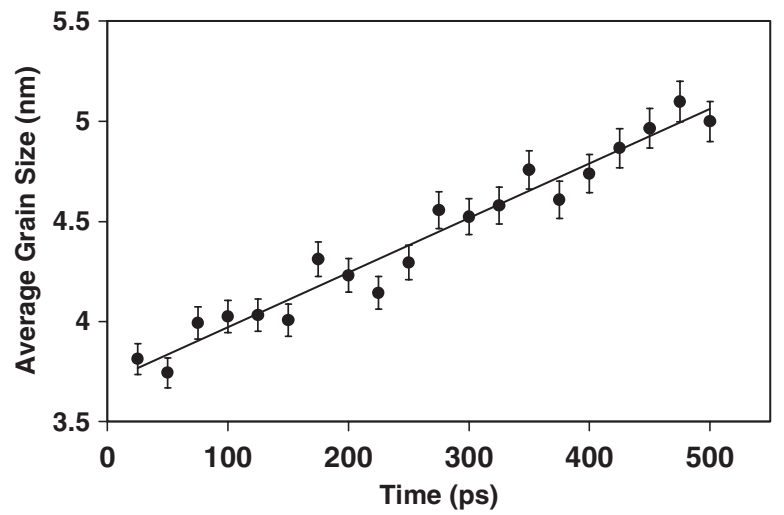

FIG. 4. Average grain size as a function of time obtained for $1300 \mathrm{~K}$. 


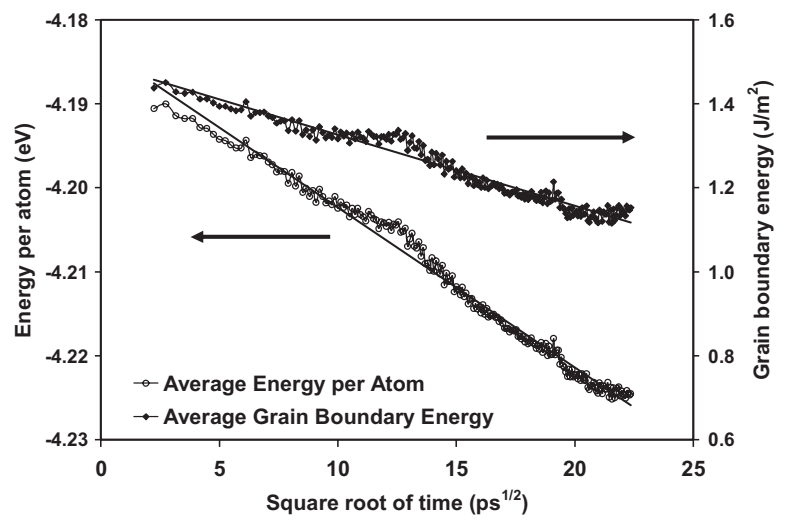

FIG. 5. Average block energy per atom and grain boundary energy as a function of time at $1300 \mathrm{~K}$.

grain growth proceeds, there will be $N$ grains with an average volume $v=V / N$. If the grains are considered cubic in shape, this means that the average area of each grain is $6[V / N]^{2 / 3}$. The total area of grain boundary is then $A=3 V^{2 / 3} N^{1 / 3}$. In this geometry, $N=V / d^{3}$, where $d$ is the grain size, and the total area is then $A=3 V / d$. Alternatively, if the grains are considered spherical, the area of each grain is $4 \pi[3 V / 4 \pi N]^{2 / 3}$, and the total area is $A=\frac{1}{2} 3^{2 / 3}[4 \pi]^{1 / 3} V^{2 / 3} \quad N^{1 / 3}$. In this geometry, $N=$ $6 V / \pi d^{3}$, and, in terms of $d$, the total grain boundary area is again $A=3 \mathrm{~V} / \mathrm{d}$. We use this relation to estimate the total grain boundary area for grains of arbitrary shape. Note that this estimate treats the outer boundary of our simulation cell as a grain boundary, and, in the limit of only one grain in the system, it gives the total grain boundary area as one-half the outer area of our simulation cube of volume $V$. The total energy of the grain boundaries contained in the sample was obtained as $E_{\mathrm{gb}}=n\left(E-E_{\mathrm{pl}}\right)$, where $n$ is the total number of atoms in the sample and $E_{\mathrm{pl}}$ is the energy of the perfect lattice at the simulation temperature. Finally, the average grain boundary energy per unit area can be calculated as $\gamma=E_{\mathrm{gb}} / A$. The results are included in Fig. 5

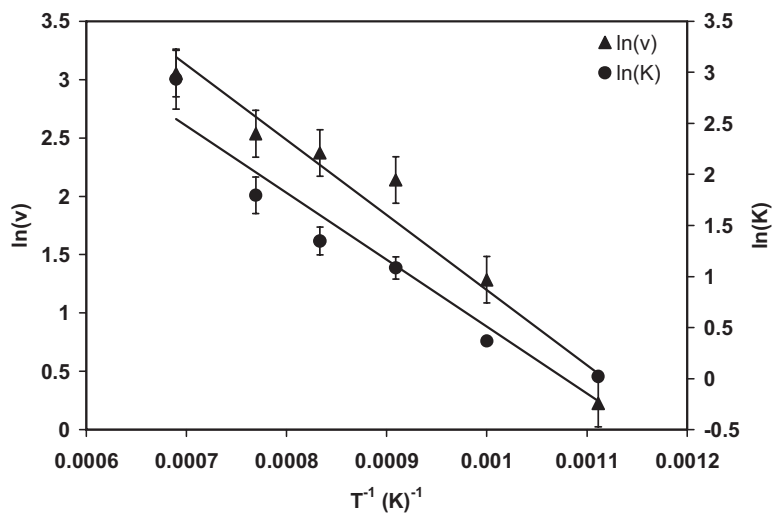

FIG. 6. Arrhenius plot of the average grain boundary velocities $(v)$ and parabolic constants in the energy evolution $(K)$ for the treatments at various temperatures. for the grain boundary energy evolution during the treatment at $1300 \mathrm{~K}$. The initial grain boundary energy obtained at this temperature is $1.4 \mathrm{~mJ} / \mathrm{m}^{2}$, again in excellent agreement with the values obtained by Mendelev et al. [16] using the same potential. As the simulation proceeds, the average grain boundary energy per unit area decreases significantly to $1.1 \mathrm{~J} / \mathrm{m}^{2}$. During the treatment time $(500 \mathrm{ps})$, the number of grains in the sample decreased from the initial 15 to 6 . The average grain size increased from 3.7 to $4.5 \mathrm{~nm}$. The decrease in grain boundary energy per unit area does not occur during the initial transient period, but rather it occurs during the entire process, in which the grain size increased by over $20 \%$ and the total number of grains decrease to about half the original number. This occurs through a process of simultaneous grain boundary migration and grain rotation. Table II shows the rotation angle for the center grain of the sample as a function of the annealing time. The table shows rotation angles around the $z$ and $x$ axes and values up to 6 degrees were observed. These results suggest that significant grain rotation accompanies the observed decrease in average grain boundary energy. Based on a simple model employing a stochastic theory, Moldovan et al. [17] investigated the coarsening of a two-dimensional polycrystalline microstructure due solely to a grain-rotation coalescence mechanism. Their work shows that the growth exponent is critically dependent on the rotation mechanisms. The rate of grain boundary energy decrease with rotations observed in our results is of the same order of magnitude as that reported by Moldovan et al. [18] based on simulations in Pd. We obtain a rate of $2.44 \mathrm{~J} / \mathrm{m}^{2} \mathrm{rad}^{-1}$, which can be compared to their reported value of $4.5 \mathrm{~J} / \mathrm{m}^{2} \mathrm{rad}^{-1}$. Moldovan et al. [18] also present a theory of diffusionaccommodated grain rotation. Applying the equations they derived to our case, we obtain a rate of rotation around the $z$ axis of $1.6 \times 10^{8} \mathrm{rad} / \mathrm{s}$. This can be compared with the rotation rate we observe from the data in Table II, which is $2.7 \times 10^{8} \mathrm{rad} / \mathrm{s}$.

We now turn to a more detailed analysis of the reasons for the linear kinetics observed. Assuming that the grain boundary curvature-induced driving force decreases with increasing grain size, the grain boundary mobility should constantly increase to keep the migration velocity constant. Figure 7 shows the calculated grain boundary mobility assuming the standard curvature driven driving force and an average velocity of $12.639 \mathrm{~m} / \mathrm{s}$ found in our analysis (Table I) for $1300 \mathrm{~K}$. The figure shows a linear dependence of the mobility on the inverse grain size. The mobility increase found in our simulations as the grains grow cannot be attributed to the fact that the average grain boundary energy decreases, because low energy, low-angle, and special boundaries are usually less mobile than high-angle random boundaries. Our result therefore implies a significant size effect, with the mobility of boundaries constrained by nanometer grain sizes being lower than that of equivalent boundaries in macroscopic-size grains. We postulate that the size effect is due to the fact that the 
TABLE II. Rotation angles around the $z$ and $x$ axes observed for the central grain in the simulation at $1300 \mathrm{~K}$.

\begin{tabular}{cccccccr}
\hline \hline Annealing time (ps) & \multicolumn{1}{c}{5} & \multicolumn{1}{c}{50} & 100 & 250 & 325 & \multicolumn{1}{c}{425} & 500 \\
\hline Rotation around the $z$ axis (degrees) & 1.70 & 2.77 & 2.69 & 5.66 & 5.48 & 6.60 & 3.11 \\
Rotation around the $x$ axis (degrees) & -0.86 & -2.23 & -3.43 & -0.34 & 0.19 & -0.97 & -2.12 \\
\hline \hline
\end{tabular}

boundary regions near the triple junctions have a decreased mobility. This assumption is similar to that proposed by Zhou, Dang, and Srolovitz [19] in a recent study of size effects on grain boundary mobility in thin films. In the thin film geometry, the driving force for migration was maintained constant, and the velocity of a single planar boundary was found to increase with film thickness. Zhou, Dang, and Srolovitz [19] attribute the mobility decrease for thinner films to the fact that the boundary regions near the surface have a lower mobility. A simple model can be constructed along the same lines, assuming that the mobility of the boundary in regions near the triple junctions is lower. This model predicts that the mobility should follow the form [19] $M=M^{\infty}[1-m / d]$, where $d$ is the grain size and $M^{\infty}$ is the grain boundary mobility for macroscopically sized grains. Figure 7 shows the results of fitting our data to this equation, and we obtain $M^{\infty}=30.2 \mathrm{~m} \mathrm{~s}^{-1} \mathrm{Gpa}^{-1}$ and $m=2.78 \mathrm{~nm}$. Our results are consistent with this simple model for the size effect in the mobility of grain boundaries for nanosized grains. The value of $m$ obtained represents the range of the effect of triple junctions on grain boundary mobility. For thin films, Ref. [19] reported a similar dependence of the mobility of a planar asymmetric $\Sigma=5$ boundary on film thickness using the same interatomic potentials at $1200 \mathrm{~K}$ [19] with values $M^{\infty}=$ $135 \mathrm{~m} \mathrm{~s}^{-1} \mathrm{GPa}^{-1}$ and $m=1.11 \mathrm{~nm}$.

In summary, our results show that, for $4 \mathrm{~nm}$ grain size nanocrystals, linear grain growth kinetics are observed, contrary to the well-known square root of the time kinetics observed in coarse-grained counterparts. The linear grain growth can be attributed to the fact that the mobility of nanosized grain boundaries are size-dependent and significantly increase as the grains grow. The growth process for

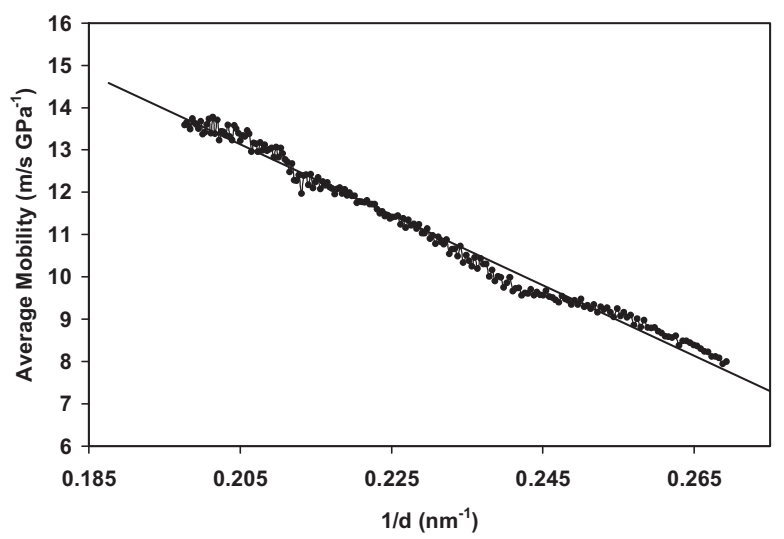

FIG. 7. Average grain boundary mobility as a function of time for the treatment at $1300 \mathrm{~K}$. these very small sizes is accompanied by grain rotation and a decrease in the average grain boundary energy per unit area. The activation energy obtained for the process coincides with that observed for grain boundary selfdiffusion in general boundaries. The mobility of the grain boundaries increases with grain size, and the linear grain growth kinetics is consistent with a simple model for the size effect on the mobility.

This work was supported by NSF, Materials Theory and performed in System X, Virginia Tech's teraflop computing facility. We acknowledge helpful discussions with A. Rollet, E. Holm, M. Mendelev, and E. Bringa. Thanks to S. Plimpton for providing LAMMPS.

[1] J.R. Weertman, D. Farkas, K. Hemker, H. Kung, M. Mayo, R. Mitra, and H. Van Swygenhoven, MRS Bull. 24, 44 (1999).

[2] J. Schiotz and K. Jacobsen, Science 301, 1357 (2003).

[3] J. Schiotz, T. Vegge, F. Tolla, and K. Jacobsen, Phys. Rev. B 60, 11971 (1999).

[4] J. Schiotz, Scr. Mater. 51, 837 (2004).

[5] H. Van Swygenhoven, M. Spaczer, A. Caro, and D. Farkas, Phys. Rev. B 60, 22 (1999).

[6] H. Van Swygenhoven, A. Caro, and D. Farkas, Mater. Sci. Eng. A 309-310, 440 (2001).

[7] D. Farkas and W. A. Curtin, Mater. Sci. Eng. A 412, 316 (2005).

[8] A. J. Haslam, D. Moldovan, V. Yamakov, D. Wolf, S. R. Phillpot, and H. Gleiter, Acta Mater. 51, 2097 (2003).

[9] C. S. Pande and R. A. Masumura, Mater. Sci. Eng. A 409, 125 (2005).

[10] M. Hourai, P. Holdway, A. Cerezo, and G. D. W. Smith, Mater. Sci. Forum 386-3, 397 (2002).

[11] A. J. Haslam, S. R. Phillpot, H. Wolf, D. Moldovan, and H. Gleiter, Mater. Sci. Eng. A 318, 293 (2001).

[12] C. E. Krill, L. Heflen, D. Michels, H. Natter, A. Fitch, O. Masson, and R. Birringer, Phys. Rev. Lett. 86, 842 (2001).

[13] A. F. Voter and S. F. Chen, Mater. Res. Soc. Symp. Proc. 82, 175 (1987).

[14] S. J. Plimpton, J. Comput. Phys. 117, 1 (1995).

[15] H. Van Swygenhoven, D. Farkas, and A. Caro, Phys. Rev. B 62, 831 (2000).

[16] M. I. Mendelev, H. Zhang, and D. J. Srolovitz, J. Mater. Res. 20, 1146 (2005).

[17] D. Moldovan, V. Yamakov, D. Wolf, and S. Phillpot, Phys. Rev. Lett. 89, 206101 (2002).

[18] D. Moldovan, D. Wolf, and S. R. Phillpot, Acta Mater. 49, 3521 (2001).

[19] L. Zhou, H. Dang, and D. J. Srolovitz, Acta Mater. 53, 5273 (2005). 